\title{
Commonwealth Fund International Health Policy Survey: Befragung zur Grundversorgung
}

Seit 2010 nimmt die Schweiz am internationalen Health Policy Survey der amerikanischen Stiftung «Commonwealth Fund» teil. In diesem Jahr standen erstmals seit der Teilnahme der Schweiz die Grundversorgerinnen und Grundversorger im Fokus.

Esther Kraft ${ }^{a}$, Markus Weber ${ }^{b}$, Sarah Vilpert ${ }^{c}$

$\begin{array}{ll}\text { a } & \text { FMH } \\ \text { b } & \text { BAG } \\ \text { c } & \text { Obsan }\end{array}$

Zur besseren Lesbarkeit wird wenn nicht ausdrücklich erwähnt - die männliche Schreibweise verwendet.

* USA, Deutschland, Niederlande, Kanada, Grossbritannien, Frankreich, Australien, Norwegen, Neuseeland. Die Daten zum ebenfalls teilnehmenden Schweden lagen leider zur Zeit der Redaktion dieses Textes noch nicht vor.
Korrespondenz:

FMH, Abteilung Daten,

Demographie und Qualität

Elfenstrasse 18

CH-3000 Bern 15

Tel. 0313591111

Fax 0313591112

ddq[at]fmh.ch
Die Grundversorgung ist in vielen Ländern von hoher Aktualität. Der Commonwealth Fund [1] stellt deshalb in dreijährigem Rhythmus die Grundversorgerinnen und Grundversorger ins Zentrum. Beim Commonwealth Fund handelt es sich um eine gemeinnützige amerikanische Stiftung, die seit 1998 jährlich international vergleichende gesundheitspolitische Befragungen durchführt. Das Bundesamt für Gesundheit BAG und die FMH führten gemeinsam die diesjährige Befragung der Grundversorger in der Schweiz durch. Die meisten Fragen wurden den Grundversorgerinnen und Grundversorgern aller teilnehmenden Länder* gestellt. In Zusammenarbeit mit «Hausärzte Schweiz» wurden für die Schweiz einige Fragen ergänzt, die sich speziell auf die hiesige Situation beziehen.

Ein unabhängiges externes Befragungsinstitut interviewte 1025 Grundversorger in der Schweiz (606 Deutschschweiz, 294 Romandie und 124 Tessin). 73\% der Befragten sind zwischen 45 und 64 Jahre alt, der Anteil der Frauen liegt bei 30\%, jener der Männer bei 70\%. Die Stichprobe entspricht damit der Grundgesamtheit der Grundversorgerinnen und Grundversorger in der Schweiz. Rund ein Drittel arbeiten in einer Stadt (37\%) oder in einem Vorort einer Stadt (39\%) und ein Fünftel in einem grösseren Ort (25\%).

Die Fragen des Commonwealth Fund International Health Policy Survey, ergänzt durch die erwähnten landesspezifischen Ergänzungen, betrafen eine Vielzahl von Themen wie z.B. Zufriedenheit mit dem Gesundheitssystem, Zugang zu den Gesundheitsleistungen, Zusammenarbeit mit anderen ärztlichen und medizinischen Leistungserbringern. Die nachfolgenden Ausführungen sind ein Auszug aus den umfassenden Resultaten. Bei der Interpretation der internationalen Daten müssen die länderspezifischen Eigenschaften des jeweiligen Gesundheitssystems mit berücksichtig werden.

\section{Schweizer Resultate im internationalen Vergleich}

Hohe Zufriedenheit der Ärzte mit ihrer Tätigkeit im internationalen Vergleich

Die Zufriedenheit der 1025 befragten Grundversor-

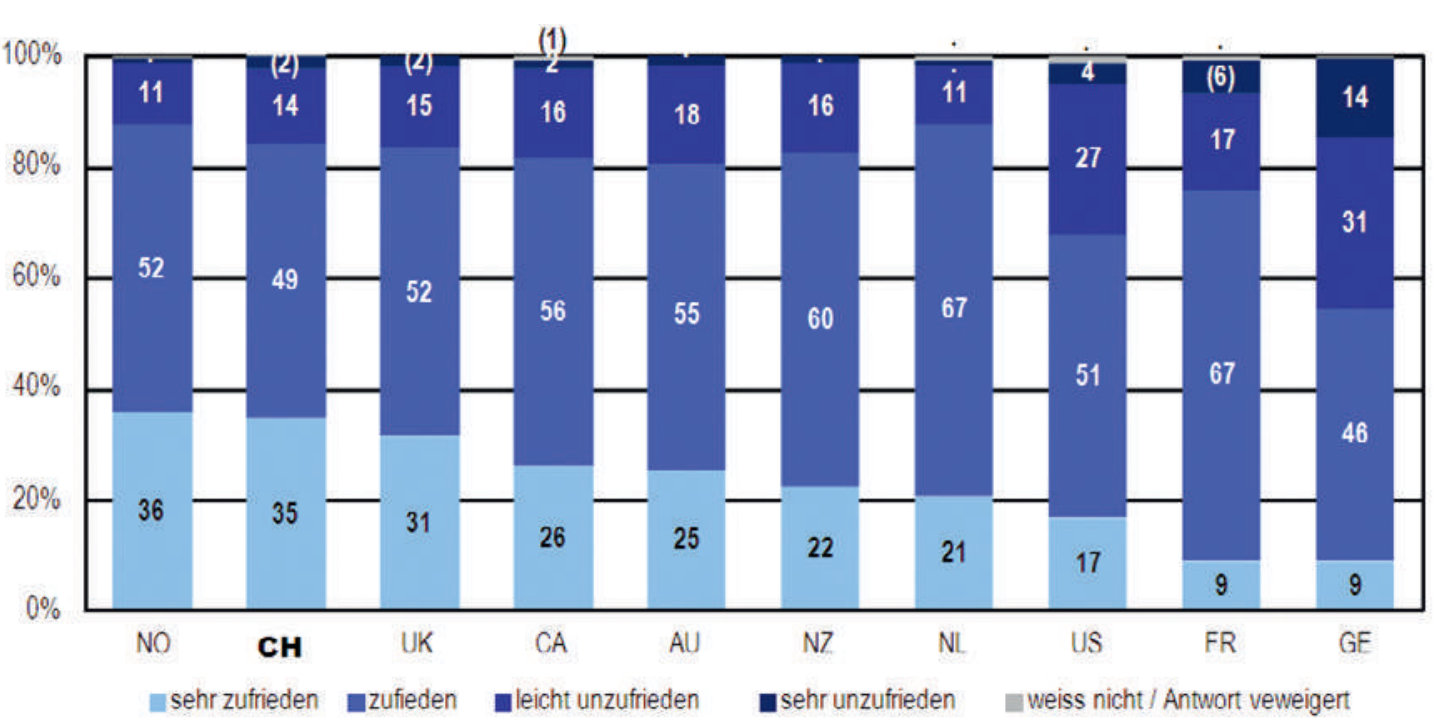

Abbildung 1

Zufriedenheit der Grundversorger mit ihrer ärztlichen Tätigkeit. Antworten von 0-9 Personen und Zahlen in Klammern haben eingeschränkte statistische Aussagekraft $(\mathrm{n}=10-29)$. Quelle: The Commonwealth Fund, International Health Policy Survey 2012. 
ger mit ihrer ärztlichen Tätigkeit ist im internationalen Vergleich hoch (Abb. 1). Zufrieden oder sehr zufrieden sind 84\% der Grundversorger. Dies widerspiegelt sich noch deutlicher in Bezug auf die Ausund Weiterbildung: 92\% stufen sich als zufrieden oder sehr zufrieden ein. 88\% der Interviewten geben an, mehr als 55 Stunden pro Woche zu arbeiten, was den zweiten Platz im internationalen Vergleich bedeutet; nur in Frankreich wird mehr gearbeitet. Bei der Interpretation gilt $\mathrm{zu}$ beachten, dass beispielsweise die Grundversorger in Frankreich weniger Patienten behandeln als in der Schweiz. Über $90 \%$ bezeichnen den zeitlichen Aufwand für administrative
Einkommen aber mit jenem der Spezialisten verglichen, sinkt die Zufriedenheit (17\%).

\section{Patienten haben Zugang zu medizinischen Leistungen}

Im internationalen Vergleich nimmt die Schweiz beim Zugang zu medizinischen Leistungen eine Spitzenposition ein. Der grösste Teil der Patienten (88\%) erhält ohne Mühe spezielle Diagnosetests und muss nicht oder selten auf Behandlungen warten (84\%). Fast alle (62\%) oder die meisten (26\%) der Patienten erhalten einen Termin am selben oder am nächsten Tag bei ihrem Hausarzt, wenn sie es wünschen. Dies

\section{Die in der Schweiz tätigen Grundversorger sind im internationalen Vergleich mit ihrer ärztlichen Tätigkeit zufrieden.}

Tätigkeiten im Zusammenhang mit den Krankenkassen oder den Abrechnungen als Problem. Für etwas weniger als die Hälfte der Grundversorger (46\%) funktioniert das Gesundheitssystem recht gut, und es sind nur kleine Änderungen nötig, dass es besser funktioniert, was im internationalen Vergleich einem mittleren Rang entspricht. Für die andere Hälfte (49\%) braucht es grundsätzliche Änderungen. Zwei Drittel der Befragten geben an, dass in den letzten drei Jahren die Qualität der medizinischen Versorgung der Patienten etwa gleich geblieben ist. Der Umfang der medizinischen Versorgung für die Patienten ist für etwas mehr als die Hälfte (59\%) der Interviewten in etwa richtig. Von den befragten Grundversorgern geben 57\% an, mit ihren Einkommen zufrieden oder sehr zufrieden zu sein. Wird das liegt auch daran, dass die meisten Ärzte Zeit reservieren, um Termine am selben Tag anbieten zu können (81\%), und auch angeben, neue Patienten aufzunehmen (86\%). Rund vier Fünftel der Ärzte (78\%) haben in ihrer Praxis eine Regelung, die es Patienten erlaubt, auch ausserhalb der Sprechstunde einen Arzt oder eine MPA aufzusuchen.

\section{Grundversorger begleiten Patienten engmaschig} Der grosse Teil der befragten Grundversorger macht Hausbesuche (90\%), unterstützt und koordiniert die Versorgung der Patienten nach dem Spitalaufenthalt (96\%), bezieht bei Bedarf die sozialen Dienste oder die Spitex ein (98\%) und berät die Patienten u. a. bei der Einnahme von Medikamenten (100\%). Eine vergleichbar hohe Unterstützung erhalten nur Patienten

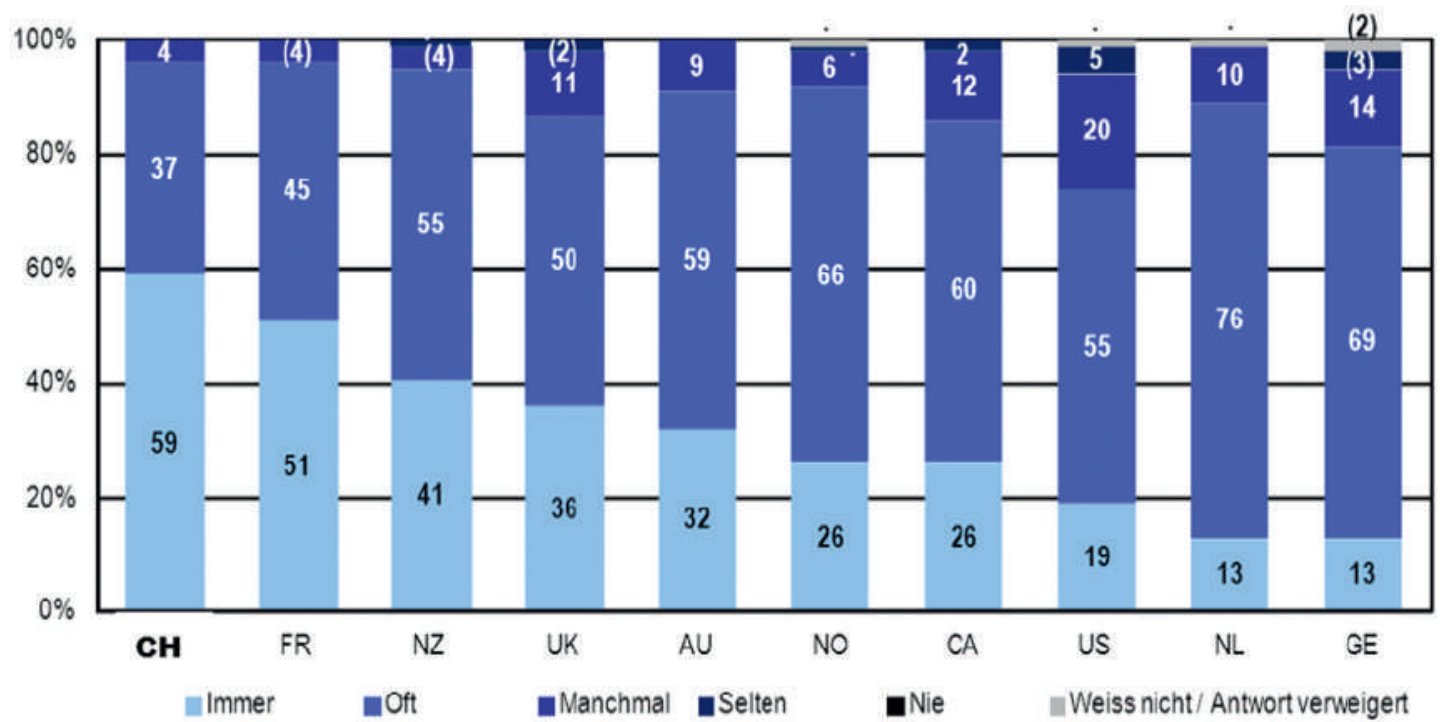

Abbildung 2

Erhalt eines Berichts vom Spezialisten mit allen relevanten Gesundheitsinformationen. Antworten von 0-9 Personen und Zahlen in Klammern haben eingeschränkte statistische Aussagekraft $(n=10-29)$. Quelle: The Commonwealth Fund, International Health Policy Survey 2012. 
in Grossbritannien. Bei der Betreuung von Patienten mit schweren chronischen Erkrankungen arbeiten die Grundversorger eng mit der Spitex zusammen (62\%) und eher selten mit eigenem Personal, das spezifisch für Patienten mit schweren chronischen Erkrankungen da ist (6\%). Die meisten Grundversorger sind zufrieden oder sehr zufrieden mit der Zeit, die sie pro Patient einsetzen können (69\%).

\section{Spitzenplatz der Schweiz bei Zusammenarbeit zwischen Grundversorgern und Spezialisten} 96\% der Grundversorger erhalten vom Spezialisten immer oder oft alle relevanten Gesundheitsinformationen (Abb. 2), 87\% der Befragten werden ebenso informiert über Änderungen, die der Spezialist bei den Medikamenten oder bei der Behandlung vorgenommen hat.

\section{Praxis-Informationssysteme sind noch wenig verbreitet}

Weniger als die Hälfte der Befragten (41\%) arbeiten mit elektronischen Patientendossiers, was im internationalen Vergleich den letzten Platz bedeutet. Dies widerspiegelt sich darin, dass die Grundversorger z.B. Schwierigkeiten haben, Patientenlisten nach Diagnosen, Labortestergebnissen oder Medikamenten zu erstellen. Im Vergleich mit den anderen teilnehmenden Ländern liegt die Schweiz diesbezüglich auf den hinteren Rängen. England, Australien und Neuseeland teilen sich bei diesen Themen die Spitzenpositionen.

\section{Die Teilnahme an Qualitätszirkeln ist sehr verbreitet:}

\section{$82 \%$ der Grundversorger nehmen daran teil.}

\section{Qualitätszirkel werden rege genutzt}

Etwas mehr als ein Drittel (37\%) der Grundversorger gleicht die eigenen ärztlichen Leistungen mindestens einmal pro Jahr mit Zielvorgaben ab. Lediglich Norwegen weist diesbezüglich einen tieferen Wert aus. England, Neuseeland und die USA sind diesbezüglich Spitzenreiter. Ihre Praxis zertifiziert hat ein Viertel. Hingegen sind rund 60\% der Befragten im Bilde, wie die ärztliche Leistung ihrer Praxis im Ver- gleich zu anderen Praxen abschneidet. Die Teilnahme an Qualitätszirkeln ist sehr verbreitet: $82 \%$ der Grundversorger nehmen daran teil, die meisten davon einmal pro Monat. Gemäss den befragten Grundversorgern werden in den Praxen Daten in Bezug auf klinische Ergebnisse (12\%) und Patientenzufriedenheitsbefragungen (15\%) noch eher selten regelmässig empfangen und genutzt.

\section{Einzelpraxen und ärzteeigene Netzwerke}

Mehr als die Hälfe (56\%) der Grundversorger in der Schweiz arbeitet in einer Einzelpraxis. Der Anteil der Einzelpraxen ist im internationalen Vergleich hoch, nur in Frankreich ist er noch höher. Bei den Gruppenpraxen in der Schweiz zeigt sich, dass in rund 60\% dieser Praxen Ärzte mit gleichen Fachrichtungen tätig sind und meistens zwei bis vier Ärzte (79\%) dort arbeiten. 61\% der Befragten sind einem Ärztenetzwerk angeschlossen, die ärzteeigenen Netzwerke sind mit $87 \%$ deutlich in der Überzahl.

\section{Schlussbemerkung}

Die in der Schweiz tätigen Grundversorger sind im internationalen Vergleich mit ihrer ärztlichen Tätigkeit zufrieden. Ebenso beurteilen sie den Zugang der Patienten zu den medizinischen Leistungen als gut. Die Schweiz nimmt eine Spitzenposition bei der $\mathrm{Zu}$ sammenarbeit zwischen Grundversorgern und Spezialisten ein, und auch die Betreuung der Patienten ist gut. Die Praxisinformationssysteme und auch die Gruppenpraxen sind im Vergleich mit den anderen Ländern noch wenig verbreitet.

Diese Ausführungen stellen lediglich einen Auszug aus den umfassenden Resultaten des Commonwealth Fund International Health Policy Survey 2012 dar. Das Schweizerische Gesundheitsobservatorium Obsan hat auch dieses Jahr wiederum einen detaillierten Bericht mit weiteren Auswertungen veröffentlicht. Den vollständigen Bericht finden Sie unter www.obsan.ch und auf der Internetseite des BAG [2].

Das BAG und die FMH stehen gerne für Auskünfte zur Verfügung (BAG: media[at]bag.admin.ch oder 031 3229505 / FMH: ddq[at]fmh.ch oder 03135911 11).

\footnotetext{
Referenzen

1 www.commonwealthfund.org

2 www.bag.admin.ch/themen/gesundheitspolitik/ 11625/index.html?lang=de
} 